\title{
The 4-month-old infant's perception of holograms and real objects: A demonstration of equivalence
}

\author{
CHARLES A. NELSON \\ Purdue University, West Lafayette, Indiana \\ and \\ PHILIP SALAPATEK \\ Institute of Child Development, University of Minnesota, Minneapolis, Minnesota
}

\begin{abstract}
Three experiments were conducted to determine whether 4-month-old infants perceive and respond to holograms as real objects. Infants in the first experiment failed to differentiate between a hologram of a toy car and the real car from which the hologram was constructed. In a second and third experiment, infants responded to holograms of two objects as they did to the real objects. The implications of using holograms in the study of early perceptual development are discussed.
\end{abstract}

Considerable attention has been directed in recent years to studying the infant's response to the human face (for relevant reviews, see Cohen, DeLoache, \& Strauss, 1979, and Fagan, 1979). The vast majority of these studies have used photographs of faces as stimuli, although real faces have been used occasionally (e.g., Barrera \& Maurer, 1981; Carpenter, 1974; Dirks \& Gibson, 1977; Haith, Bergman, \& Moore, 1977; Maurer \& Salapatek, 1976). The decision by most investigators to use photographic stimuli has typically rested on the technical advantages inherent in such stimuli-that is, the degree of control one maintains over the relevant dimensions of the stimulus that may affect perception, and the ability to present the same stimulus on repeated trials with great precision. Such attributes are not present in real faces. Thus, even though it is the perception of real faces one ultimately hopes to understand, most investigators have elected to use photographs, with the hope that such findings would generalize to how real faces were perceived. Unfortunately, it has not yet been demonstrated that infants perceive real faces and photographs of faces as equivalent, and therefore, this assumption may be questioned.

Recently, Nelson and Horowitz (1983) and Nelson (in press) have suggested that holograms may prove to be a

The research was supported by an NICHD postdoctoral fellowship (1F32 HD 06231) to the first author, and by grants to the University of Minnesota, Center for Research in Human Learning (NSF/BNS-7722075 and HD-01136). The advice and facilities made available by Richard Peterson of Bethel College are gratefully acknowledged, as is the technical assistance and shop facilities made available by Mervyn Bergman. A special debt of thanks is also extended to Gerald Wasserman, who commented on an earlier version of the manuscript. Philip Salapatek died unexpectedly on March 4, 1984. Reprint requests should be sent to the first author at the Department of Psychological Sciences, Peirce Hall, Purdue University, West Lafayette, IN 47907. more valid and useful means of studying the infant's response to the human face than either photographs or real faces. Three arguments were invoked to support this claim. First, Nelson and Horowitz (1983) reported that 5-month-old infants appeared to respond to holograms of faces much the way infants respond to real faces (e.g., Kreutzer \& Charlesworth, 1973), but qualitatively differently from the way infants respond to photographs of faces (e.g., Young-Browne, Rosenfeld, \& Horowitz, 1977). Second, other investigators using comparably aged infants have reported that infants can discriminate between real, three-dimensional objects and photographs of these objects (Rose, 1977, Experiment 1), and that recognition memory is better for three-dimensional objects than for photographs of these objects (Ruff, Kohler, \& Haupt, 1976). Finally, holograms share with photographs the ability to control the stimulus from trial to trial, yet are very similar optically to real objects. These arguments collectively suggest that holograms may provide an alternative to real objects when stimulus control is of concern, and photographs when ecological validity is of concern.

However reasonable the above claims are for using holograms as a substitute for either real objects or photographs, there has not yet been a test of whether infants perceive real objects and holograms of these objects as equivalent, or of whether holograms are perceived as categorically distinct from photographs. The present investigation was designed to address the first issue; that is, do infants respond in the same way to holograms of objects as they do to real objects?

Holography is a three-dimensional imaging technique that uses laser light to record the patterns of light waves reflected from an object onto the emulsion of lightsensitive film. If the hologram perfectly reconstructed the 
light wave front emitted by the original object, then a hologram would contain all of the information about the object's size, shape, position, and reflectivity that was imparted by the object itself (Jenkins \& White, 1976). However, in practice, certain physical differences do exist between a hologram and the object that produced it. These differences are that: (1) the image may appear semitransparent (unless, as in the case in the present experiments, an opaque screen is placed behind the film), (2) the texture of the hologram is typically grainier than that of the real object (due in part to the type of laser used and the film development process), (3) the color of the hologram may differ from that of the real object (i.e., the color depends on the chromaticity of the light source used to view the hologram), and (4) the field of view of a hologram is intrinsically limited in direction and extent. From the vantage point of the perceptual psychologist, the features holograms share most with real objects are that they are both three-dimensional and in perspective (i.e., small changes in eye/head position result in a slightly different view of the object, such as seeing one object hidden behind another).

Low-power, helium-neon $(\mathrm{HeNe})$ lasers are typically used to construct holograms of stationary stimuli. Because of their low power, relatively long exposure times are necessary to record the image (e.g., 2 to $10 \mathrm{sec}$ ). Accordingly, any stimulus that moves, however slightly, creates a blurred image. To construct holograms of moving objects generally requires a much more powerful laser, such as a ruby-pulse laser. Because of their high power, much shorter exposure times are possible, thereby allowing one to construct holograms of nearly any object, be it moving or stationary. It has been estimated, for example, that the largest allowable shift of an object toward or away from the film emulsion during the exposure period is approximately $.08 \mu$. Given that the burst of light produced by a ruby pulse laser rarely exceeds much more than 3 $\times 10^{-8} \sec (.03 \mu \mathrm{sec})$, even an object moving at $2 \mathrm{~m} / \mathrm{sec}$ would move only $.06 \mu$, well within the tolerable limit (Klein, 1970). It should be apparent, however, that this narrow range of permissible movement precludes using low power lasers for any but the most stable of objects. Given that the only laser available to the investigators was a low-power HeNe laser, it was necessary to limit the holograms we constructed to stationary stimuli. It was for this reason that inanimate objects were used in the present set of studies.

As alluded to above, holograms contain much of the information contained in a real object. This attribute, coupled with the fact that holograms provide as much stimulus control as do photographs, suggests that holograms might be an ideal stimulus in studies of infant perception, particularly when the ecological validity of the stimulus was of concern. However, there has not yet been a test of whether holograms are perceived by infants as equivalent to real objects. Such a test would be important, since subtle differences (such as those outlined above) do in fact exist between holograms and real objects. Accordingly, if infants perceive holograms and real objects as equivalent, they would be doing so on the basis of either having ignored such differences or having been unable to discriminate such differences. The present set of studies was designed to examine both issues. Specifically, would 4month-old infants be able to discriminate between a real object and a hologram of that object, and would they, in general, respond to holograms as they did to real objects?

\section{EXPERIMENT 1}

\section{Method}

Subjects. In the first experiment, 48 full-term 4-month-old infants ( $\mathrm{M}$ age $=122.31$ days) served as subjects. All infants were recruited from birth announcements in the local paper and were tested at the University of Minnesota.

Stimuli. Transmission holograms of a toy car and a baby bottle were constructed by the authors, although only the car stimulus was used in the first experiment. These stimuli were chosen for their similar size, brightness, and luminance, and because it was desirable to use common, familiar objects. Both holograms were constructed using an Aerotech 15-mW HeNe laser (Model L516) and high-resolution Agfa Gevaert holographic plate film (8E75HD-NAH-5 $\times 4$, unb). The film was exposed for $5 \mathrm{sec}$ and then developed and fixed. The film itself measured $10.2 \times 12.0 \mathrm{~cm}$, and served essentially as an aperture behind which appeared the holographic image. The image itself appeared approximately $25 \mathrm{~cm}$ behind the film plane, which corresponded to how far the real object was positioned behind the film during the actual construction of the hologram. Infants were seated approximately $60 \mathrm{~cm}$ from the film plane, resulting in the greatest subtense of both the real car and hologram of the car to be approximately $21^{\circ}$; both the real bottle and the hologram of the bottle subtended approximately $18^{\circ}$. An attempt was made to match both stimuli (i.e., car and bottle) and both stimulus media (i.e., hologram and real object) for luminance. Thus, the luminances of the hologram of the car, real car, hologram of the bottle, and real bottle were, respectively, $58,36,30$ and $46 \mathrm{~cd} / \mathrm{m}^{2}$. None of these reflectance differences were discriminable by adults. The holograms were projected using an $18-\mathrm{W}$ sodium lamp that radiates most of its energy at $580 \mathrm{~nm}$. It was placed approximately $1.5 \mathrm{~m}$ behind the film plane at a $45^{\circ}$ angle. The nature of the light source thus resulted in both holograms' appearing "yellow." In the conditions in which the real object was to be viewed, infants looked through an unexposed, but fixed, piece of holographic film, behind which the real objects (toy car or baby bottle) could be viewed (to match for visual angle, both objects were positioned on a stand approximately $25 \mathrm{~cm}$ behind the film plane). To ensure that the real object was of the same chromaticity and luminance as its holographic counterpart, the real object was both rear illuminated by the sodium lamp and front lighted by white light passed through a Kodak Wratten No. 3 gelatin filter (i.e., the dominant wavelength of light passed by this filter was $580 \mathrm{~nm}$ ).

As stated earlier, holograms and real objects share many optical properties, although some differences do exist. In order to establish the nature of such similarities and differences, many of the parents of the infants tested in the study were informally questioned about their perception of the stimuli. While these subjects ( $N=$ approximately 30 ) were invariably unable to tell apart the hologram and real object (the stimuli were presented one at a time, and although formal data were not collected, responding appeared to be at chance level), they did indicate that they thought there was a difference between the two. After compiling these subjects' responses, the differences noted tended to revolve around texture differences. Specifically, the hologram tended to appear "grainier," and the real object tended to appear "shinier." Thus, although adult subjects were unable to determine whether the stimulus was a real object or a hologram, they could nonetheless detect differences between the two. The question posed of infants was essentially 
the same; that is, would infants be able to detect the difference between the hologram and real object, and would they respond to the holograms of the objects as they had to the real objects?

Procedure. In all three experiments, the infants were placed in a car seat approximately $60 \mathrm{~cm}$ from a gray Masonite screen, on the back of which was mounted the hologram or the blank piece of holographic film. Dark blue curtains shielded the infants' peripheral vision on both sides, and a similar curtain was placed directly behind the stand upon which the real objects were viewed. (This curtain provided a backdrop against which both the hologram and real object could be viewed.) Infants thus looked through the holographic film to see either the real object or the holographic image. A weak infrared light source positioned above and to the right illuminated the infant's face. A video camera containing a Uvicon tube and a zoom lens was positioned above and in line with the infant's face and projected an image of the infant from the neck up onto a TV screen. Observers trained to a mean level of $91 \%$ reliability and blind to condition recorded the infants' looking times from the TV. All infants were tested with the room lights dimmed.

A fixed-trial habituation procedure was employed, in which infants received six 10-sec familiarization trials of one stimulus, followed by two 10-sec trials of either a different stimulus (experimental infants) or the same stimulus (control infants). Each trial was timed from the beginning of each look, thereby ensuring that all infants were indeed attending at the beginning of a trial. After each trial, a gray shutter (the same color as the Masonite screen) was slid over the opening, signaling the end of the trial. The intertrial interval was approximately $3 \mathrm{sec}$. The number of familiarization and test trials and the duration of each trial were derived in pilot work.

Design. All infants were assigned randomly to four groups. One group was habituated to the hologram of the car and tested on the real car; a second group saw the reverse (experimental groups). A third and a fourth group saw only the hologram of the car or the real car during both the familiarization and test phases (control groups). An increase in looking from the last familiarization trial to the test trials on the part of the experimental infants relative to no such increase on the part of the control infants was taken as evidence of discrimination. If, as expected, infants perceived the hologram as a real object, then no such recovery should be evident in any group.

\section{Results}

Since we were not concerned with the habituation phase of the experiment (i.e., our goal in using a fixed-trial procedure was simply to ensure that all infants were familiarized to the standard stimulus, not necessarily that they had habituated to the stimulus, and it had been indicated in pilot work that the length and number of familiarization trials was sufficient to facilitate discrimination), only the discrimination data were analyzed. Looking times served as the dependent measure. To test for response recovery, the last trial of the familiarization phase (preshift) was compared with the average of the two Phase 2 trials (referred to in the figure as postshift). This repeated measures variable was used to compare the two orders comprising the experimental groups (i.e., habituate to the hologram of the car, test on real car or the reverse) with the two orders comprising the control groups. An ANOVA revealed no significant main effects or interaction effects of any order or group. (For all comparisons, $p>1$ ). In addition, as can also be seen by the error bars in Figure 1, there were no differences in looking times to the hologram of the car vs. real car on any trial. Thus, for example, infants habituated to the real car in the experimental group looked no more and no less than those habituated to the real car or the hologram of the car in either the experimental or the control groups. Simi-

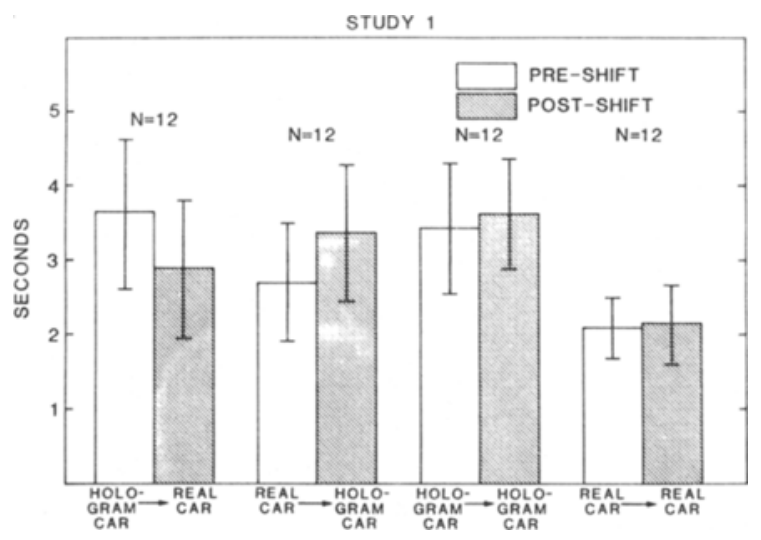

Figure 1. Duration of time infants spent looking on the preshift trial (Trial 6) and the postshift trials for each of the two experimental and two control conditions in Experiment 1. Error bars represent the standard error of the mean. Overlapping error bars on comparison histograms indicate no significant difference in looking times.

larly, infants tested on the real car in the experimental groups looked no more and no less than those infants tested on the hologram of the car or the real car in either the experimental or control groups. (For all comparison, $\mathrm{p}>1)$.

\section{Discussion}

From these results, it appeared that infants were unable to discriminate the real car from the hologram of the car. However, this conclusion was based essentially on the lack of response recovery. Because of the difficulty in interpreting results based on the failure to reject the null hypothesis $(\mathrm{Ho})$, a second and third experiment were conducted. The goal here was to ensure that the habituation paradigm employed was sensitive to detecting psychologically significant differences. Should such differences not be detected, then one must question the validity of the conclusions drawn from the first experiment. On the other hand, should such differences be detected, these conclusions would be strengthened.

\section{EXPERIMENT 2}

If holograms and real objects are perceived as identical (i.e., give rise to the same perceptual experience), it follows that for any two real objects that are discriminable, holograms made of these two objects should also be discriminable. This prediction, of course, rests on two assumptions: (1) The objects used must indeed be discriminable, and (2) the paradigm employed must be sensitive to detecting such discrimination. The goal of the second experiment was to test the discriminability of the two real objects, using the same paradigm as in Experiment 1 . The goal of the third experiment was to extend these findings to holograms made of the real objects.

\section{Method}

In the second experiment, another group of 4-month-old infants ( $\mathrm{N}$ $=48$ ), chosen as before, served as subjects ( $\mathrm{M}$ age $=124.5$ days). 
The experimental infants were habituated to either the real car and tested on the real bottle, or the reverse; the control infants saw either the real car or the real bottle for both phases of the study. ${ }^{1}$

\section{Results}

Looking times from the preshift phase to the postshift phase again served as the dependent measure. When the data were analyzed as in Experiment 1, the results revealed no significant main effects. However, a significant interaction effect emerged between the repeatedmeasures component and the order in which the stimuli were presented $[F(1,44)=8.88, p<.005]$. Subsequent post hoc analyses indicated that only infants habituated to the bottle and tested on the car evidenced a significant recovery of looking $(\mathrm{p}<.05)$. These results can be seen in Figure 2. As indicated by the error bars in Figure 2, it is also apparent that there was no significant difference in how long infants looked at the bottle or the car during comparable trials (e.g., infants' looking times to the bottle during the preshift phase in the experimental group was comparable to their looking times to the bottle during the preshift phase of the control group; $p>1$ ).

\section{Discussion}

From these results, it was possible to conclude that infants were able to discriminate the two objects, although this discrimination was constrained by the order in which the stimuli were presented. It is not clear what caused the stimulus presentation order effect. One possible explanation may be that one object was more interesting to look at than the other. This can be partially ruled out, however, when one examines the error bars in Figure 2. Inspection of the data indicates that there was no significant difference between how long infants looked at one object vs. another as long as the comparison was restricted to the same trial (e.g., preshift or postshift). Thus, the order effect was most likely not due to differential stimulus salience. Independent of the cause of the stimulus presentation order effect, of more immediate concern were the implications this order effect had for Experiment 3. It may

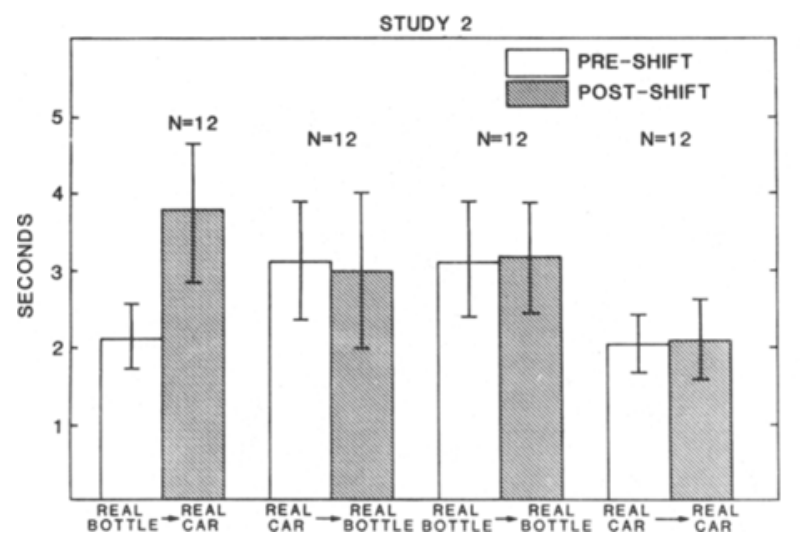

Figure 2. The data from Experiment 2. (The plotting of the data is the same as in Figure 1.) be recalled that the goal of the third experiment was to extend the findings of the second experiment to holograms of the two objects. Additionally, it was predicted that whatever results obtained with the real objects would generalize to the results obtained with the holograms of the objects, if in fact the two were perceived as identical. The prediction for the third experiment, then, was that if Experiment 2 was repeated with holograms used in place of the real objects, the results, including the stimulus presentation order effect, should be the same.

\section{EXPERIMENT 3}

\section{Method}

The third experiment was identical to the second, except that another group of 4-month-old infants $(\mathrm{N}=48)$ was tested ( $\mathrm{M}$ age $=126.98$ days) and holograms were used in place of the real objects. ${ }^{2}$

\section{Results}

The results, which were analyzed as in the first two experiments and can be seen in Figure 3, yielded a significant repeated-measures $\times$ stimulus order interaction $[F(1,44)=10.38, p<.005]$. Post hoc analyses indicated that only infants familiarized to the bottle hologram and tested on the car hologram evidenced a significant recovery of looking $(\mathrm{p}<.01)$.

\section{Discussion}

Overall, the results of the third experiment were identical to those of the second. The two stimuli were again found to be discriminable, although this discrimination was again constrained by the order in which the stimuli were presented. It thus appears that young infants do, indeed, perceive and respond to holograms as they do to real objects.

The results of the third experiment perfectly replicated those of the second, including the stimulus presentation order effect. It is still not clear what caused this order effect, however. There is some hint from the data that the car (both hologram and real) recruited more looking

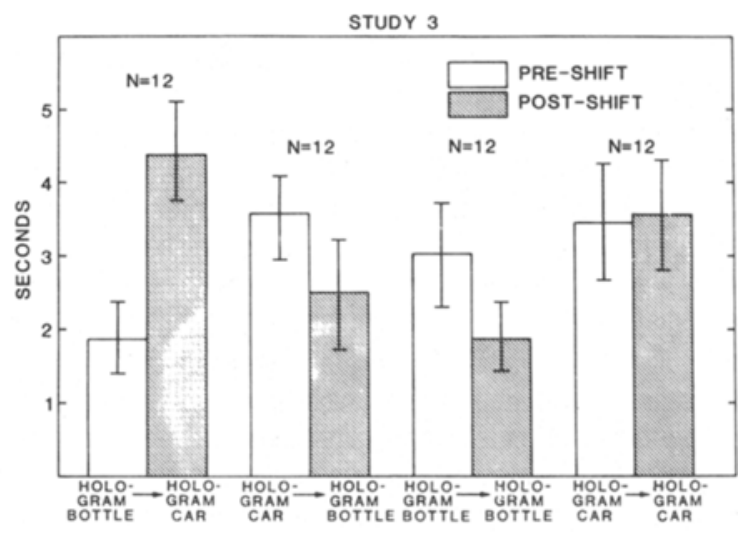

Figure 3. The data from Experiment 3. (The plotting of the data is the same as in Figure 1.) 
than the bottle (both hologram and real), although these suspicions were not borne out statistically. Thus, initial stimulus salience must again be ruled out as an explanation. Nevertheless, independent of this order effect, the major finding of note is that this order effect replicated from Experiment 2 to Experiment 3, reinforcing the claim that holograms are perceived as real objects.

\section{GENERAL DISCUSSION}

The findings from all three experiments can be summarized as follows. When 4-month-old infants are presented with a hologram of a car and a real car, they fail to distinguish between them. When infants this age are asked to discriminate between holograms of two different objects, they behave as another group of infants had when asked to discriminate between the two real objects from which the holograms were constructed. Collectively, from both experiments, there is the strong suggestion that 4-month-old infants respond equivalently to holograms and to real objects in a discrimination paradigm. ${ }^{3}$

One potential limitation of the present set of experiments was the habituation paradigm employed. While our goal was not necessarily to habituate infants to one stimulus or another but rather simply to familiarize them to these stimuli, one might still find fault with the study for not ensuring that infants had indeed habituated. In addition, the conclusions drawn from the first experiment were based on a failure to reject $\mathrm{Ho}$, a point of contention for many investigators. While both criticisms cannot be summarily dismissed, it can be argued that both criticisms are unwarranted in the present context. First, it has been demonstrated that habituation is a sufficient, although not necessary, condition for discrimination (e.g., Olson \& Sherman, 1983). Thus, whether or not infants habituated to the standard during the familiarization phase has little bearing on whether infants discriminated the new stimulus during the test phase. However, if one fails to ensure that all infants have habituated to the standard (which is next to impossible using a fixed trial procedure) but nevertheless interprets findings based on the failure to reject $\mathrm{Ho}$, then it is incumbent upon the investigator(s) to demonstrate that the paradigm employed is sensitive to detecting psychologically significant differences. In the present study, this was done in Experiment 2 and replicated in Experiment 3. In both experiments, infants discriminated the car from the bottle (real or hologram), although evidence of the reverse was not obtained. While it is possible (although not verifiable) that the stimulus presentation order effect was a result of insufficient familiarization with the car (i.e., it may take longer for infants to encode a car stimulus than a bottle stimulus, for whatever reason), this is beyond the goal of the present set of studies-namely, that the paradigm employed was sensitive to detecting an infant's ability to discriminate between two discriminable stimuli, and that this held for both real objects and holograms of these objects. As such, the study demonstrates that (1) infants could not distinguish between a real car and a hologram of this car, and
(2) infants responded to the hologram-bottle vs. hologramcar discrimination task as they had to the real-bottle vs. real-car discrimination task. Collectively, the results provide convincing evidence that 4-month-old infants respond equivalently to holograms and real objects.

What are the implications of the above result? First, given (1) the evidence that infants respond differently to two-dimensional vs. three-dimensional objects (e.g., Fagan, 1979; Rose, 1977; Ruff et al., 1976); (2) the present evidence that real objects and holograms are perceived equivalently; (3) the fact that holograms share many of the technical advantages of photographs; and (4) the fact that there are just as few restrictions placed on viewing holograms as there are on viewing photographs (e.g., in both cases, infants can move around only to a limited degree without compromising the validity of the dependent measure, and in both cases infants either look through or at an aperture to view the stimuli), it seems reasonable to accept the claim that holograms can be used as a substitute for both photographs and real objects. This suggestion, of course, would most apply in situations in which the real objects themselves could not be used, such as real faces, due to the inability to control and precisely manipulate the stimulus.

The above argument suggests that holograms are an experimentally valid substitute for both real objects and twodimensional representations of these objects when the situation warrants. However, there is yet another situation in which it might be preferable to use holograms. This concerns the ability to ask questions that are not possible to ask using other stimulus media. For example, holograms made of moving objects (i.e., holographic stereograms) allow one to induce motion in an otherwise static stimulus by having the observer move his/her head and eyes. Thus, using such stimuli it would be possible to examine active perceptual search and the extent to which an infant may or may not be able to regulate and control visual information in the environment. Additionally, nearly all types of holograms could be used to study infants' manual exploration of visible but intangible objects (e.g., does such incongruity present a conflict to the infant?). With further advances in the medium, other uses could undoubtedly be found.

Overall, the present set of studies suggests that 4-monthold infants perceive and respond to holograms of inanimate objects as they do to real objects, at least within the confines of the discrimination procedure employed. The utility of such stimuli will most likely be found in studies in which it is desirable to use as realistic a stimulus as possible without trading off stimulus control, and in studies in which other stimulus media are inadequate for addressing the question of interest.

\section{REFERENCES}

Barrera, M. E., \& Maurer, D. (1981). Discrimination of strangers by the three-month-old. Child Development, 52, 203-206.

CARPEnter, G. (1974). Visual regard of moving and stationary faces in early infancy. Merrill-Palmer Quarterly, 20, 181-194.

Cohen, L. B., DeLoache, J. S., \& Strauss, M. S. (1979). Infant visual 
perception. In J. Osofsky (Ed.), Handbook of infancy (pp. 393-437). New York: Wiley.

DiRKs, J., \& GiBSON, E. J. (1977). Infants' perception of similarity between live people and their photographs. Child Development, 48 , 124-130.

FAGAN, J. F. (1979). The origins of facial pattern recognition. In M. Bornstein \& W. Kessen (Eds.), Psychological development from infancy (pp. 83-113). Hillsdale, NJ: Erlbaum.

Haith, M. M., Bergman, T., \& Moore, M. J. (1977). Eye contact and face scanning in early infancy. Science, 198, 853-855.

JenkINS, F. A., \& WHITE, H. E. (1976). Fundamentals of optics. New York: McGraw-Hill.

KLEIN, H. A. (1970). Holography. New York: Lippincott.

Kreutzer, M. A., \& Charlesworth, W. R. (1973, March). Infants' reactions to different expressions of emotion. Paper presented at the biennial meetings of the Society for Research in Child Development, Philadelphia.

MAURER, D., \& SAlAPATEK, P. (1976). Developmental changes in the scanning of faces by young infants. Child Development, 47, 523-527.

Nelson, C. A. (in press). The perception and recognition of facial expressions in infancy. In T. Field \& N. Fox (Eds.), Social perception in infancy. Norwood, NJ: Ablex.

Nelson, C. A., HoRowitz, F. D. (1983). The perception of facial expressions and stimulus motion by 2 - and 5 -month-old infants using holographic stimuli. Child Development, 54, 868-877.

Olson, G. M., \& Sherman, T. (1983). Attention, learning and memory in infants. In M. M. Haith \& J. J. Campos, (Eds.), Handbook of child psychology (Vol. 3, pp. 1001-1080). New York: Wiley.

Rose, S. A. (1977). Infants' transfer of response between twodimensional and three-dimensional stimuli. Child Development, 48 , 1086-1091.

RufF, H. A., Kohler, C. J., \& HAUPT, D. L. (1976). Infant recognition of two- and three-dimensional stimuli. Developmental Psychology, 12, 455-459.
Young-Browne, G., Rosenfeld, H. M., \& Horowitz, F. D. (1977). Infant discrimination of facial expressions. Child Development, 48 , 555-562.

\section{NOTES}

1. The control group infants from Experiment 1 who had seen only the real car provided the data for this control group in Experiment 2. No statistically significant differences emerged when the duration of time infants from the different control groups spent looking on the preshift and postshift trials, and the preshift trial vs. the postshift trial were compared. It was therefore inferred that the infants were, in fact, from the same general population of subjects (and behaved as would be expected for control subjects), thereby validating their inclusion into the data analysis of the second experiment.

2. The control group infants from Experiment 1 who had seen only the hologram of the car provided the data for this control group in Experiment 3. An analysis comparable to the one referenced in footnote 1 was performed, and the same conclusions were drawn.

3. It should be noted that these findings may be extended to a broader age range than that studied in the present investigation. We also tested, informally, many of the 2- to 5-year-old siblings of our infant subjects. We did so by handing them the car in the testing room with the lights on, and then showing them the hologram of the car with the lights dimmed. When asked if the two cars were the same or different, children unanimously replied "same" (approximate $n=15$ ). This evidence is, of course, anecdotal, but should serve to reinforce and possibly extend the findings reported herein.

(Manuscript received March 9, 1984; revision accepted for publication July 23,1984 .) 